\title{
Radiological Analysis of Sagittal and Cross- sectional Morphology of Congenital Lumbar Spinal Stenosis
}

\section{Qihui Cheng}

The Third Affiliated Hospital of Southern Medical University

\section{Ganghui Yin}

The Third Affiliated Hospital of Southern Medical University

\section{Minjun Huang}

The Third Affiliated Hospital of Southern Medical University

\section{Haojie Mi}

The Third Affiliated Hospital of Southern Medical University

\section{Junwei Guan}

The Third Affiliated Hospital of Southern Medical University

\section{Sishu Yin}

The Third Affiliated Hospital of Southern Medical University

\section{Zhongmin Zhang}

Nanfang Hospital

Bo Yan ( $\sim$ yanbosmu@gmail.com)

The Third Affiliated Hospital of Southern Medical University

\section{Research Article}

Keywords: Congenital lumbar spinal stenosis, Spine and pelvis parameters,Sagittal plane, cross-sectional morphological

Posted Date: July 26th, 2021

DOl: https://doi.org/10.21203/rs.3.rs-734824/v1

License: (c) (1) This work is licensed under a Creative Commons Attribution 4.0 International License. Read Full License 


\title{
Radiological analysis of sagittal and cross-sectional morphology of congenital lumbar spinal stenosis
}

\author{
Qihui Cheng*1, Ganghui Yin*1, Minjun Huang ${ }^{1}$, Haojie Mi ${ }^{1}$, Junwei \\ Guan $^{1}$, Sishu Yin ${ }^{2}$, Zhongmin Zhang\#³, Bo Yan\# ${ }^{1}$
}

\begin{abstract}
Background Purpose This retrospective study was applied to investigate the morphology characteristics of the spine and pelvis in patients with congenital spinal stenosis, to explore the effect of morphological parameters in the pathogenesis and development of the disease.
\end{abstract}

Methods The analysis is based on data of a case-control study, including 40 patients (19 females/21 males) with congenital lumbar spinal stenosis, 40 patients (17females/23males) with age- Sex- and the waist and leg pain score-matched acquired lumbar spinal stenosis and 40 age- Sex-matched normal volunteers(controls). Lumbar MRI, lumbar computerized tomography (CT) and full-length radiographs were used to obtain sagittal and cross-sectional parameters. Parameters including pelvic incidence (PI), sacral slope (SS), pelvic tilt (PT), lumbar lordosis (LL), sagittal vertical axis (SVA), and thoracic kyphosis (TK) on the sagittal plane were measured on full-length radiographs and analyzed. The anteroposterior (AP) bone canal diameter and spinal canal area of L4 were collected and analyzed on Lumbar CT. Lumbar MRI was taken to evaluate the angle of the ligamentum flavum at the level of L4/5 intervertebral space, and the Lumbar disc degeneration degree was calculated.

Results Total scores for lumbar disc degeneration and the angle of the ligamentum flavum were significantly lower in the congenital group than in the acquired group $(\mathrm{P}=0.02$ and $\mathrm{P}=0.012$, respectively ; $\mathrm{P}$ $<0.05$ ). The differences of LL, PT, SVA and TK values were statistically significant , while the differences of SS and PI were not significant among the acquired, congenital, and control groups. TK values were significantly lower in the congenital group than in the acquired and control groups $(\mathrm{P}=0.024$ and $\mathrm{P}=0.006$, respectively; $\mathrm{P}<$ 0.05 ). Patients in the congenital and acquired groups had significantly lower LL values than patients in the control group ( $\mathrm{p}=0.000$ and 0.041 , respectively; $\quad \mathrm{P}<0.05)$. The mean value of LL was $30.31^{\circ} \pm 13.42^{\circ}$ in the congenital group, while $41.10^{\circ} \pm 12.51^{\circ}$ in the acquired group. And the difference of LL between these two groups was statistically significant $(p=0.000<0.05)$. The SVA values of the congenital group, acquired group, and control group increased respectively(all $\mathrm{P}<0.05)$. The $\mathrm{PT}$ in the congenital group showed significantly lower values than 
the acquired group $\quad(p=0.041<0.05)$. There is no statistically significant difference in other parameters. The correlations between LL and PI are well in the congenital group（ $\mathrm{r}=0.336 ; \mathrm{P}=0.034 ）$, acquired group（ $\mathrm{r}=0.464$; $\mathrm{P}=0.003$ ） and control group ( $\mathrm{r}=0.584 ; \mathrm{P}=0.000)$. However, the trend line of LL/PI in the acquired group was drawn below the control population. Also, the trend line of LL/PI in the congenital group was below the waist and leg pain score-matched acquired group with lower lumbar degeneration.

Conclusion In addition to bony structural stenosis, the smaller angle of the ligamentum flavum may be an anatomical factor that causes the smaller effective area of the spinal canal in patients with congenital lumbar spinal stenosis. Patients with congenital lumbar spinal stenosis show a significant reduction in the physiological curvature of the thoracic and lumbar spine, and the trunk leans forward. In addition to intervertebral disc degeneration and pain factors, bony spinal stenosis is also a possible factor leading to smaller LL in patients with congenital lumbar spinal stenosis. LL less than $41^{\circ}$ can be used as the initial screening standard for congenital lumbar spinal stenosis among patients with lumbar spinal stenosis.

\section{Keywords Congenital lumbar spinal stenosis ; Spine and pelvis parameters; Sagittal plane;} cross-sectional morphological

\section{Background}

Lumbar spinal stenosis is one of the most common diseases which needs surgical intervention in the elderly, and is also one of the most likely causes of low back pain in patients aged over $50^{1}$. Based on its etiology, Arnoldi et al. have classified LSS as congenital (developmental), acquired (degenerative) (75\%), or a combination of both $^{2}$.The most specific symptom of lumbar spinal stenosis, whether it is congenital lumbar spinal stenosis or acquired lumbar spinal stenosis, was first illustrated by Verbiest in 1954 , which is neurogenic claudication including soreness, numbness, weakness, and heaviness of the lower limbs after walking a long distance ${ }^{3,4}$. Even though the clinical symptoms of the two are similar, they have different pathogenesis and treatment methods. If the segment of congenital lumbar spinal stenosis is not decompressed during the operation, it will lead to a higher risk of reoperation. So patients with congenital lumbar spinal stenosis may need to preventively decompress the stenotic segment. Therefore, it is very necessary to identify congenital lumbar spinal stenosis from acquired lumbar spinal stenosis ${ }^{4-6}$. Some scholars claimed that acquired lumbar spinal stenosis was caused by IVD degeneration, trauma, medical sources, etc. While developmental spinal stenosis is more likely a genetic disturbance due to impaired lumbar vertebrae development in fetal and postnatal ${ }^{7}$. Patients with congenital lumbar spinal stenosis generally have a low threshold for neural compression due to a pre-existing narrowed spinal canal ${ }^{8}$. Compared to patients with acquired lumbar spinal stenosis, congenital lumbar spinal stenosis occurs earlier in age with similar clinical findings but with multilevel involvement and fewer degenerative changes. In this group of people, minor degeneration can cause severe clinical symptoms. The sagittal balance of the human body is coordinated and maintained by the spine and pelvis. The ideal spine and pelvis alignment allows individuals to maintain a standing posture with minimal energy consumption ${ }^{9-12}$. In the past, clinicians only focused on the physiological curvature and disease characteristics of the spine. In recent years, the clinical significance of the spine and pelvis has been deeply studied. Duval-Beaupere et al first reported that PI (pelvic incidence) represents 
the normal sagittal pelvic morphology in $1992^{13}$. Many scholars have found that spinal sagittal pelvic shape not only plays an important role in maintaining the body sagittal balance but is also involved in the pathogenesis of isthmic spondylolisthesis and other diseases. Xi Yang et al studied that lower PI may promote the occurrence of lumbar degenerative diseases. After disc degenerative change, the acquired structural and compensatory factors would bring about a more vertical spine. At present, there are few studies on the different types of patients with congenital lumbar spinal stenosis' sagittal spine-pelvic morphology. This study compares the sagittal spine-pelvic parameters of developmental lumbar spinal stenosis, acquired lumbar spinal stenosis, and normal controls, and further explores the sagittal spine-pelvic morphological characteristics of patients with developmental lumbar spinal stenosis.

\section{Materials and methods}

\subsection{Healthy subjects and patients}

This retrospective study selects patients with lumbar spinal stenosis who were diagnosed by two senior spine surgeons, hospitalized for surgical treatment in our department, and had complete imaging data during January 2017 and October 2020. Axial CT images of the patients have been examined and the patients have been grouped as congenital and acquired lumbar spinal stenosis. Patients with an anteroposterior (AP) bone canal diameter less than $12 \mathrm{~mm}$ had been considered as DLSS ${ }^{14,15}$. The diagnosis of all patients was confirmed by medical history, physical examination, and imaging examination. Patients who have previously received lumbar spine surgery or combined with lumbar spondylolisthesis, trauma, tumor, and bilateral femoral head disease were excluded in this study. In the end, 40 patients with congenital lumbar spinal and 40 age-,Sex- and low back pain score-matched patients with acquired lumbar spinal stenosis were included in this study. At the same time, 40 normal people matching the age and sex ratio of the lumbar spinal stenosis group were recruited as the control group. Every subject was conscious of that the data might be used to study and publish. All of them over 18 years old signed an informed consent form upon admission, and this study was approved by the institutional ethics committee of our hospital.

\subsection{Visual Analogue Scale (VAS)}

The pain is represented by 11 numbers from 0 to 10,0 means no pain, and 10 means the most pain. The higher the score, the stronger the pain. It was recommended to use the following cut-off points on painful VAS: $\leq 2,3-5,6-8,8-10$, Representing no pain, mild pain, moderate pain, and severe pain. According to the individual's pain experience, the subjects pointed out the degree of pain that suits them on the ruler at the time of admission.

\subsection{Radiological assessment}

All subjects' full-length radiographs were taken in the neutral position at admission and were measured the following indicators. Two independent measurements of the above sagittal spine-pelvic parameters were conducted by the same researcher, and the mean of parameters was calculated.

1.3.1 Spine parameters(Fig1 A): All the spine parameters are masured as described below ${ }^{16,17}$. (1) The LL (lumbar lordosis) was defined as the Cobb angle between the upper endplate of the L1 and S1. (2) The 
TK(thoracic kyphosis): The angle between the tangent to the upper endplate of the T5 cone and the tangent to the lower endplate of the T12 cone. (3) SVA(sagittal vertical axis): The horizontal distance between the vertical line of $\mathrm{C} 7$ and the upper back edge of the sacrum. If the vertical line of $\mathrm{C} 7$ just passes through the upper posterior corner of the sacrum, it is extremely 0 , indicating that the sagittal plane of the spine is in a balanced state; if the vertical line of $\mathrm{C7}$ is located behind the upper posterior corner of the sacrum, it is recorded as a negative value, indicating that the sagittal plane of the spine is in a state of balance. A Negative balance means that the trunk is tilted backward; if the $\mathrm{C} 7$ vertical line is in front of the upper posterior corner of the sacrum, it is recorded as a positive value, which means that the sagittal plane of the spine is in positive balance and the trunk is tilted backward.

1.3.2 Pelvic parameters (Fig1 B ) : All the pelvic parameters are masured as described below, ${ }^{9,19}$ (1) The PI (pelvic incidence) was defined as the angle between the vertical line of S1 upper endplate and the line connecting the midpoint of the sacral plate to the midpoint of the bilateral femoral head center. After adulthood, PI is a constant parameter, which can fully reflect the compensatory ability of pelvic posterior rotation, that is, PI has a limiting effect on the ability of pelvic supination ${ }^{18}$. (2) The SS (sacral slope) was defined as the angle between the tangent to the upper endplate of the sacrum and the horizontal line. (3) The PT was defined as the angle between the plumb line and the line connecting the midpoint of the sacral plate to the midpoint of the bilateral femoral head center. PT is positive when the midpoint of the upper endplate of S1 is behind the axis of the femoral head, and negative when it is anterior.SS and PT are parameters that describe the spatial position of the pelvis.

1.3.3 Cross-section parameters (Fig2) : All the parameters are masured as described below. (1) The angle of the ligamentum flavum(LF) of midline level of L4/5 intervertebral space: Made a cut line at the outer edge of the dura mater on both sides, and the angle between the two lines was measured. (2) According to the pfirrmann classification, score the total degree of L1-5 intervertebral disc degeneration of patients in the congenital and acquired groups: 1 grade means 1 point; 2 means 2 points, and so on. The total degree of degeneration is the addition of the degeneration scores of lumbar intervertebral discs at all levels. (2) According to the pfirrmann classification, score the total degree of L1-5 intervertebral disc degeneration of patients in the congenital and acquired groups: 1 grade means 1 point; 2 means 2 points, and so on. The total degree of degeneration is the summation of the degeneration scores of lumbar intervertebral discs at all levels.

\subsection{Statistical Analysis}

Two measurements of the above sagittal spine-pelvic parameters of the enrolled population were carried out by the same researcher under double-blind conditions, and the final value is expressed as the mean value of the two measurements. Statistical analysis was performed using the SPSS 20.0 statistical software. Tests for parameters were performed to decide on the appropriate statistical method. Log transformations were considered for data sets that exhibited skewed distribution. Supposing that $\log$ transformations failed to establish normal distribution, nonparametric tests were considered. If $\log$ transformations can establish a normal distribution, then the independent sample t-test is used to analyze various data. $\mathrm{P}<0.05$ indicates that the difference is statistically significant. ROC curves are drawn for LL. Classification data were performed by $\chi^{2}$ test. When comparing multiple sets of data, one-way ANOVA for parametric data was used to compare the variables. Bonferroni post hoc test was utilized for subgroup comparison after the ANOVA test. Bivariate correlation and linear regression models were established to find the factors that may affect LL. 


\section{Results}

\subsection{Patients with congenital lumbar spinal stenosis have a more upright spine and a more forward lean in the sagittal plane. (Fig3)}

Comparing the general indices between these three groups, no significant differences in age, gender were found. It showed that patients were matched in age, the gender ratio in these three groups. Also, there was no significant difference in the VAS scores of lower limb pain and low back pain between the development group and the acquired group total scores for lumbar disc degeneration and the angle of the ligamentum flavum were significantly lower in the congenital group than in the acquired group ( $\mathrm{P}=0.020$ and $\mathrm{P}=0.012$, respectively) . Through CT measurement of the lumbar spine, we can find that the Spinal canal area and anteroposterior (AP) bone canal diameter of L4 in the congenital group are smaller than the acquired group, and the difference is statistically significant. $(\mathrm{P}=0.000$, all) (Table 1)

The values of LL, PT, SVA, and TK were significantly different, while the SS and PI were not among the acquired, congenital, and control groups. (Table 2) A post hoc test (Table 3) showed that TK was significantly lower in the congenital group than in the acquired and control groups $(\mathrm{P}=0.024$ and $\mathrm{P}=0.006$ respectively $<0.05)$. Both the congenital and acquired groups had significantly lower LL than the control group $(\mathrm{p}=0.000$ and 0.041 , respectively $<0.05$ ). Also, LL was significantly lower in the congenital group than in the acquired groups $(p=0.000$ $<0.05$ ). While, the SVA of the congenital group, the acquired group, and the control group increased gradually, and the difference between every two groups showed statistically significant. (congenital-normal $\mathrm{P}=0.000<0.05$; congenital-acquired $\mathrm{P}=0.045<0.05$; acquired-normal $\mathrm{P}=0.002<0.05$ ) .In terms of $\mathrm{PT}$, the congenital group showed significantly lower values than the acquired group $(p=0.041)$. There is no statistically significant difference in other parameters.Regarding the relationship between PI and Other sagittal parameters, the correlation between LL and PI is all well in the congenital group ( $\mathrm{r}=0.336 ; \mathrm{P}=0.034)$, acquired group $(\mathrm{r}=0.464 ; \mathrm{P}=0.003$ ), and control group $(\mathrm{r}=0.584 ; \mathrm{P}=0.000)$. However, the trend line of LL/PI in the acquired group was drawn below the control population. Factors such as the degeneration of the lumbar intervertebral disc, Low back pain, and back muscle spasm may explain this phenomenon. The trend line of LL/PI in the congenital group was also significantly below the Waist and leg pain score-matched acquired group with more serious the degeneration of the lumbar intervertebral disc (Fig4). The results suggested that in addition to the degeneration of the lumbar intervertebral disc, low PI values and low back pain, and back muscle spasm, other factors may lead to such lower LL in the congenital group.

\subsection{Bony spinal stenosis leads to lower $L L$ in patients with lumbar spinal stenosis.}

We speculated that bony spinal canal size such as spinal canal area and anteroposterior (AP) bone canal diameter may be a factor affecting the LL of patients with lumbar spinal stenosis (congenital lumbar spinal stenosis and acquired lumbar spinal stenosis). Therefore we conducted bivariate correlation and linear regression models to clarify the relationships between LL of the 80 patients with lumbar spinal stenosis and spinal canal area, as well as the relationships between LL of patients with lumbar spinal stenosis and anteroposterior(AP) bone canal diameter. The result of multiple linear regression showed that the LL of patients with lumbar was influenced by bony spinal 
canal sizes such as spinal canal area $(\beta=-0.084, \quad 95 \% \mathrm{CI}: \quad-0.166,-0.002, \quad \mathrm{P}=0.044)$ and anteroposterior(AP) bone canal diameter $(\beta=4.269, \quad 95 \% \mathrm{CI}: 2.305,6.234, \quad \mathrm{P}=0.000)$.

\subsection{LL less than $41^{\circ}$ can be used as the initial screening standard for congenital lumbar spinal stenosis}

Because the LL of patients with congenital lumbar spinal stenosis was smaller than that of acquired lumbar spinal stenosis, and the difference was statistically significant $(\mathrm{P}<0.05)$. Therefore, we drew the ROC curve for $\mathrm{LL}$ and determined the optimal critical value of $41^{\circ}$ according to the maximum Youden index. The area of the ROC curve line is 0.735 . (Fig5) Youden index is 0.55 . According to this standard, parallel diagnostic experiments were performed. When the LL is less than $41^{\circ}$ on the whole spine radiograph, the sensitivity is $90 \%$ and the specificity is $65 \%$. LL less than $41^{\circ}$ can be used as the initial screening standard for congenital lumbar spinal stenosis among patients with lumbar spinal stenosis.

\section{Discussion}

Congenital lumbar spinal stenosis was first reported by Verbiest H in 1954. Clinical manifestations include low back pain and neurogenic claudication of lower limbs, radiculopathy, and motor weakness ${ }^{3}$. However, its pathogenesis is still not very clear. A genome-wide association study on the etiology of dlss showed that there are several single-nucleotide polymorphisms (SNP) are involved, which are ZNF704, LRP5, and DCC genes. The study also suggested that different genes are involved in L1-4 and L5-S1 in DLSS. Many previous studies conducted cross-sectional imaging studies on patients with congenital lumbar spinal stenosis and found that compared with acquired lumbar spinal stenosis, their pedicles were shorter, the lamina angle was smaller, and the AP value was smaller ${ }^{19}$. Due to the preexisting bone stenosis of the spinal canal, patients with congenital lumbar spinal stenosis have a lower threshold of nerve compression, which means that severe neurological symptoms can occur with much lower lumbar disc degeneration. Our study found that the angle of the ligamentum flavum in patients with congenital lumbar spinal stenosis is smaller than that in patients with acquired lumbar spinal stenosis. This suggests that the ligament angle may be another factor leading to the small effective spinal canal area in patients with congenital lumbar spinal stenosis.

The view that the sagittal shape of the spine and pelvis plays a crucial role in the occurrence and development of various lumbar degenerative diseases has been confirmed in recent studies. Junseok Bae et al showed a significant correlation between the location of disc herniation and spino-pelvic alignment. Compared to the LLD (lower lumbar disc hernation) and control groups, the ULD (upper lumbar disc hernation) group had significantly lower PI and $\mathrm{LL}^{20}$. The study indicates PI may play an indispensable role in the pathogenesis of degenerative lumbar intervertebral disc disease. Another study revealed acquired structure and compensatory factors decrease lumbar lordosis after intervertebral disc degeneration ${ }^{21}$. It is claimed that a higher PI and a smaller sacral table angle are crucial factors leading to lumbar spondylolysis in Chinese Han adults by Yin et al.

There are also studies showing that lumbar isthmic spondylolisthesis is complicated by lower $\mathrm{PI}^{22,23}$.All these studies showed that sagittal parameters were involved in the pathogenesis of lumbar degenerative diseases.PI was first proposed by Legaye et al. It can objectively reflect the anatomical positional relationship between the upper endplate of S1 and the femoral head, and reach a stable state in adulthood without being affected by subjective symptoms and changes in body position ${ }^{9}$.Previous studies have shown that the shape of the spine in low PI and low 
SS tends to be more linear, which leads to an increase in the vertical stress of the lumbar intervertebral disc, which is likely to cause early disc degeneration and herniation. Therefore, low PI may be a factor leading to lumbar disc herniation. At the same time, PI is the main risk factor for adolescent lumbar spondylolisthesis in Chinese Han adolescents. High PI means high risk ${ }^{10,23}$.In this study, the PI value of the congenital group was $48.40^{\circ} \pm 9.07^{\circ}$, the PI of the normal control group was $49.08^{\circ} \pm 11.76^{\circ}$, and the PI of the acquired group was $45.08^{\circ} \pm 9.17^{\circ}$. There was no significant difference between the three groups $(\mathrm{P}=0.168>0.05)$. It shows that the anatomical positional relationship between the upper endplate of $\mathrm{S} 1$ and the femoral head of patients with lumbar spinal stenosis is similar to that of normal people.

Many authors have been revealed that the sagittal profile was characterized by lower LL and TK, lower SS, and greater SVA ( a straight spine, vertical sacrum and an anterior displaced C7 plumb line )for patients with lumbar disc herniation. ${ }^{21,24-27}$ The results of this study showed that the average TK $\left(23.65^{\circ} \pm 10.82^{\circ}\right.$ VS $31.61^{\circ}$ $\pm 10.81^{\circ} \mathrm{P}=0.006 ; 23.65^{\circ} \pm 10.82^{\circ}$ and $\left.28.10^{\circ} \pm 11.76^{\circ} \quad \mathrm{P}=0.024\right)$ and $\operatorname{LL}\left(30.31^{\circ} \pm 13.42^{\circ} \quad \mathrm{VS} 47.60^{\circ}\right.$ $\pm 8.14^{\circ} \mathrm{P}=0.000 ; 30.31^{\circ} \pm 13.42^{\circ}$ and $\left.41.10^{\circ} \pm 12.51^{\circ} \quad \mathrm{P}=0.000\right)$ of the congenital group were significantly lower than those of the normal control group and acquired group, and sagittal trunk forward tilt of the congenital group was more obvious than that of the normal group (SVA $61.91 \mathrm{~mm} \pm 55.59 \mathrm{~mm}$ VS $7.60 \mathrm{~mm} \pm 24.99 \mathrm{~mm} \mathrm{P}=0.000$ ) and acquired group(SVA $61.91 \mathrm{~mm} \pm 55.59 \mathrm{~mm}$ VS $39.28 \mathrm{~mm} \pm 36.57 \mathrm{~mm} \mathrm{P}=0.045$ ). These abnormal changes in sagittal spine plane morphology are basically consistent with the results of the previously reported sagittal spine plane study of patients with lumbar disc herniation, indicating that patients with congenital lumbar spinal stenosis have a sagittal spine morphology similar to those with lumbar disc herniation ${ }^{27,28}$. This sagittal plane change is a combined effect of degeneration and pain compensation. The first is the degeneration of the lumbar intervertebral disc and the decrease in the height of the intervertebral disc leads to a decrease in LL. The onset of congenital lumbar spinal stenosis is the combined effect of lumbar disc degeneration and bony spinal stenosis. Under the condition that the bony area of the spinal canal is constant, the patient can further reduce the LL to expand the length of the spinal canal to achieve a larger effective volume of the spinal canal, so as to relieve neurological symptoms such as low back pain ${ }^{21,25}$.In addition, PI is also an innate anatomical factor that affects LL. A lower PI means a lower LL. The decrease in LL is followed by an increase in SVA, which means that the body leans forward. The result is an unstable center of gravity and a sagging field of vision. For this reason, the body will reduce TK and increase the cervical lordosis angle and bend the hips and knees to maintain a level of vision and a stable center of gravity.

But there is no statistically significant difference in the mean of SS among the congenital group 、 the normal control group and the acquired group. (SS $30.11^{\circ} \pm 9.56^{\circ}$ VS $32.47^{\circ} \pm 7.48^{\circ}$ VS $30.30 \pm 6.68$ $\mathrm{p}=0.345>0.05)$. PT of the congenital group is similar to the normal control group (PT $19.48^{\circ} \pm 8.11^{\circ} \mathrm{VS}$ $\left.16.41^{\circ} \pm 9.07^{\circ} \mathrm{P}=0.288>0.05\right)$. This indicates that SS and PT reduction is not important in the sagittal compensation mechanism in patients with congenital lumbar spinal stenosis. We also found that the LL of the acquired group was smaller than that of the normal control group $\left(41.10^{\circ} \pm 12.51^{\circ}\right.$ VS $47.60^{\circ} \pm 8.14^{\circ}$ $\mathrm{P}=0.041$ ), but the SVA of the acquired group was higher than that of the normal control group ( $39.28 \mathrm{~mm} \pm 36.57 \mathrm{~mm}$ VS $7.60 \mathrm{~mm} \pm 24.99 \mathrm{~mm} \mathrm{P}=0.002$ ). Other spinal and pelvic parameters were not statistically significant. This shows that in most cases of acquired lumbar spinal stenosis, the backward rotation of the pelvis and TK reduction (Thoracic spine straightened) are not enough to compensate for the loss of LL. 
Compared with age-, Sex- and low back pain score-matched acquired group with more severe lumbar disc degeneration, the changing trend of LL over PI in the congenital group was decreasing. This indicates that in addition to lower PI and low back and leg pain, other factors may play an important role in reducing the LL value. From bivariate correlation and linear regression models to clarify the relationships between LL of the 80 patients with lumbar spinal stenosis and spinal canal area and the relationships between LL of the patients with lumbar spinal stenosis and anteroposterior(AP) bone canal diameter, we found that bony spinal stenosis was an influencing factor leading smaller LL in patients with congenital lumbar spinal stenosis. The mechanism may be that the narrower the spinal canal, the stronger the pain sensitivity, and the more eager to relieve pain or that patients with congenital lumbar spinal stenosis have a Physiological smaller LL. If the patient with congenital lumbar spinal stenosis has a physiological small LL, this means that the factor of bony spinal stenosis needs to be taken into account when the lumbar spine is pre-curved. In other words, patients with congenital lumbar spinal stenosis may have a smaller lumbar pre-curved angle than patients with acquired lumbar spinal stenosis in the case of similar spine-pelvis sagittal plane parameters.

\section{Conclusion}

In addition to bony structural stenosis, the small included angle of the ligamentum flavum may be an anatomical factor that causes the small effective area of the spinal canal in patients with congenital lumbar spinal stenosis. Compared with patients with acquired lumbar spinal stenosis and normal people, patients with congenital lumbar spinal stenosis show a significant reduction in the physiological curvature of the thoracic and lumbar spine, and the trunk leans forward. In addition to intervertebral disc degeneration and pain factors, the degree of spinal stenosis is also an important factor leading to smaller LL in patients with congenital lumbar spinal stenosis. The reason may be that the narrower the spinal canal, the stronger the pain sensitivity, and the more eager to relieve pain. But it may also be because patients with congenital lumbar spinal stenosis have a smaller LL before the onset. This needs to be further confirmed by relevant prospective studies. In addition, we define a cut-off value based on the characteristics that patients with congenital lumbar spinal stenosis are generally smaller than those with acquired lumbar spinal stenosis. A LL which is less than $41^{\circ}$ can be used as the initial screening standard for congenital lumbar spinal stenosis among patients with lumbar spinal stenosis.

\section{Availability of data and materials}

The data analysed during the current study are available from the corresponding author on reasonable request.

\section{Abbreviations}

PI: Pelvic incidence

SS: Sacral slope

LL: Lumber lordosis

PT: Pelvic tilt

SVA: Sagittal vertical axis 
TK: Thoracic kyphosis

CG: The congenital lumbar stenosis group

AG: The acquired lumbar stenosis group

NG: The normal people group

LF: The ligamentum flavum

AP:Anteroposterior

CT:Computed Tomography

MRI:Magnetic Resonance Imaging

IVD:Intervertebral disc

DLSS:Degenerative lumbar spinal stenosis

ROC:Receiver operating characteristic

VAS: Visual Analogue Scale

\section{References:}

1 Otani, K. et al., Lumbar spinal stenosis has a negative impact on quality of life compared with other comorbidities: an epidemiological cross-sectional study of 1862 community-dwelling individuals. ScientificWorldJournal 2013590652 (2013).

2 Arnoldi, C. C. et al., Lumbar spinal stenosis and nerve root entrapment syndromes. Definition and classification. Clin Orthop Relat Res 4 (1976).

3 VERBIEST, H., A radicular syndrome from developmental narrowing of the lumbar vertebral canal. J Bone Joint Surg Br 36-B 230 (1954).

4 Cheung, P., Fong, H. K., Wong, C. S. \& Cheung, J., The influence of developmental spinal stenosis on the risk of re-operation on an adjacent segment after decompression-only surgery for lumbar spinal stenosis. BONE JOINT J 101-B 154 (2019).

5 Reale, F., Delfini, R., Gambacorta, D. \& Cantore, G. P., Congenital stenosis of lumbar spinal canal: comparison of results of surgical treatment for this and other causes of lumbar syndrome. Acta Neurochir (Wien) 42199 (1978).

6 Verbiest, H., Results of surgical treatment of idiopathic developmental stenosis of the lumbar vertebral canal. A review of twenty-seven years' experience. J Bone Joint Surg Br 59181 (1977).

7 Visuri, T., Ulaska, J., Eskelin, M. \& Pulkkinen, P., Narrowing of lumbar spinal canal predicts chronic low back pain more accurately than intervertebral disc degeneration: a magnetic resonance imaging study in young Finnish male conscripts. MIL MED 170926 (2005).

8 Lai, M., Cheung, P. \& Cheung, J., A systematic review of developmental lumbar spinal stenosis. EUR SPINE J 292173 (2020).

9 Legaye, J., Duval-Beaupère, G., Hecquet, J. \& Marty, C., Pelvic incidence: a fundamental pelvic 
parameter for three-dimensional regulation of spinal sagittal curves. EUR SPINE J 799 (1998).

10 Berthonnaud, E., Dimnet, J., Roussouly, P. \& Labelle, H., Analysis of the sagittal balance of the spine and pelvis using shape and orientation parameters. J SPINAL DISORD TECH 1840 (2005).

11 Korovessis, P. G., Stamatakis, M. V. \& Baikousis, A. G., Reciprocal angulation of vertebral bodies in the sagittal plane in an asymptomatic Greek population. Spine (Phila Pa 1976) 23 700, 704 (1998).

12 Kobayashi, T., Atsuta, Y., Matsuno, T. \& Takeda, N., A longitudinal study of congruent sagittal spinal alignment in an adult cohort. Spine (Phila Pa 1976) 29671 (2004).

13 Duval-Beaupère, G., Schmidt, C. \& Cosson, P., A Barycentremetric study of the sagittal shape of spine and pelvis: the conditions required for an economic standing position. ANN BIOMED ENG 20 451 (1992).

14 VERBIEST, H., Further experiences on the pathological influence of a developmental narrowness of the bony lumbar vertebral canal. J Bone Joint Surg Br 37-B 576 (1955).

15 Verbiest, H., Pathomorphologic aspects of developmental lumbar stenosis. Orthop Clin North Am 6 177 (1975).

16 Faro, F. D., Marks, M. C., Pawelek, J. \& Newton, P. O., Evaluation of a functional position for lateral radiograph acquisition in adolescent idiopathic scoliosis. Spine (Phila Pa 1976) 292284 (2004).

17 Lenke, L. G. et al., Adolescent idiopathic scoliosis: a new classification to determine extent of spinal arthrodesis. J BONE JOINT SURG AM 831169 (2001).

18 Roussouly, P. \& Nnadi, C., Sagittal plane deformity: an overview of interpretation and management. EUR SPINE J 191824 (2010).

19 Kitab, S. A., Alsulaiman, A. M. \& Benzel, E. C., Anatomic radiological variations in developmental lumbar spinal stenosis: a prospective, control-matched comparative analysis. SPINE J 14808 (2014).

20 Bae, J. et al., Radiological analysis of upper lumbar disc herniation and spinopelvic sagittal alignment. EUR SPINE J 251382 (2016).

21 Yang, X. et al., The characteristics of spinopelvic sagittal alignment in patients with lumbar disc degenerative diseases. EUR SPINE J 23569 (2014).

22 Yin, J. et al., Differences of Sagittal Lumbosacral Parameters between Patients with Lumbar Spondylolysis and Normal Adults. Chin Med J (Engl) 1291166 (2016).

23 Zhao, J., Xiao, Y., Zhai, X., Chen, Z. \& Li, M., Difference of Sagittal Alignment between Adolescents with Symptomatic Lumbar Isthmic Spondylolisthesis and the General Population. Sci Rep 810956 (2018).

24 Roussouly, P., Gollogly, S., Berthonnaud, E. \& Dimnet, J., Classification of the normal variation in the sagittal alignment of the human lumbar spine and pelvis in the standing position. Spine (Phila Pa 1976) 30346 (2005). 
25 Barrey, C., Jund, J., Noseda, O. \& Roussouly, P., Sagittal balance of the pelvis-spine complex and lumbar degenerative diseases. A comparative study about 85 cases. EUR SPINE J 161459 (2007).

26 Rajnics, P., Templier, A., Skalli, W., Lavaste, F. \& Illes, T., The importance of spinopelvic parameters in patients with lumbar disc lesions. INT ORTHOP 26104 (2002).

27 Endo, K., Suzuki, H., Tanaka, H., Kang, Y. \& Yamamoto, K., Sagittal spinal alignment in patients with lumbar disc herniation. EUR SPINE J 19435 (2010).

28 Fei, H., Li, W. S., Sun, Z. R., Ma, Q. W. \& Chen, Z. Q., Analysis of Spino-pelvic Sagittal Alignment in Young Chinese Patients with Lumbar Disc Herniation. ORTHOP SURG 9271 (2017).

\section{Acknowledgements}

None.

\section{Funding}

This work was supported by Science and Technology Program of Guangzhou(201904010056), National Natural Science Foundation of China( 81902172), Natural Science Foundation of Guangdong Province(2020A1515010162).

\section{Author information}

\section{Affiliations}

1. Department of Orthopedics, The Third Affiliated Hospital, Southern Medical University, Academy of Orthopedics, Guangdong Province, Guangzhou, China

2. Department of Ultrasonography, The Third Affiliated Hospital of Southern Medical University, Guagnzhou, China

3. Division of Spine Surgery, Department of Orthopadics, Nanfang Hospital, Southern Medical University, Guangzhou, China

\section{Contributions}

*Qihui Cheng, Ganghui Yin contributed equally to this work.

Qihui Cheng, Ganghui Yin and Bo Yan wrote the main manuscript text. Qihui Cheng and Minjun Huang prepared figures and tables. Haojie Mi, Qihui Cheng, and Sishu Yin performed statistics analysis. Junwei Guan and Zhongmin Zhang collected patients 
information. Bo Yan and Zhongmin Zhang surpervised the whole experiments. All authors reviewed the manuscript.

\#Corresponding author:

Correspondence to Zhongmin Zhang:nfzzm@163.com; Bo Yan: yanbosmu@gmail.com.

\section{Ethics declarations}

\section{Ethics approval and consent to participate}

This research was approved by the institutional ethics committee of The Third Affiliated Hospital of Southern Medical University. Written informed consent was obtained from all patients. All methods were carried out in accordance with relevant guidelines and regulations.

\section{Consent for publication}

Patients signed informed consent regarding publishing their data.

\section{Competing interests}

The authors declare that they have no competing interest.

\section{Figure legends}

Figure1

Ilustration of the conventional radiological parameters of the spine-pelvis on the sagittal plane.The pelvis parameters include pelvic incidence(PI), pelvic tilt(PT), and sacral slope(SS) from picture A . The spine parameters include lumbar lordosis(LL), thoracic kyphosis(TK) and sagittal vertical axis(SVA) from picture B .

\section{Figure2}

Illustration of the way to measure the angle of the ligamentum flavum (LF) of midline level of L4/5 intervertebral space. 


\section{Figure3}

Comparison of sagittal plane morphology of the CG, AG, NG groups. Arranged from left to right are the whole spine X-rays of patients with congenital lumbar spinal stenosis, acquired lumbar spinal stenosis and normal people. Compared with acquired lumbar spinal stenosis patients group and normal people group, the spine of patients with congenital lumbar spinal stenosis is more vertical and the trunk is more forward.

\section{Figure4}

Correlation between PI and LL of normal group ( NG, red label), acquired group ( AG, blue label) and congenital group ( $\mathrm{CG}$, green label).The red line, blue line, and green line increase sequentially, which means that the LL/PI trend line of the control group, acquired group, and control group gradually rises.

\section{Figure5}

ROC curve of the LL for preliminary screening of patients with congenital lumbar spinal stenosis. The area under the curve (AUC) is :0.735. ROC: receiver operating characteristic.

Table captions

Table 1: Normal information in the $\mathrm{CG}, \mathrm{AG}$, and NG.

Table 2 Comparisons of spinopelvic parameters in the CG, AG, and NG.

Table 3 Parameters comparison results of the Bonferroni post hoc test between $\mathrm{CG}, \mathrm{AG}$ and NG. 


\section{Figures}

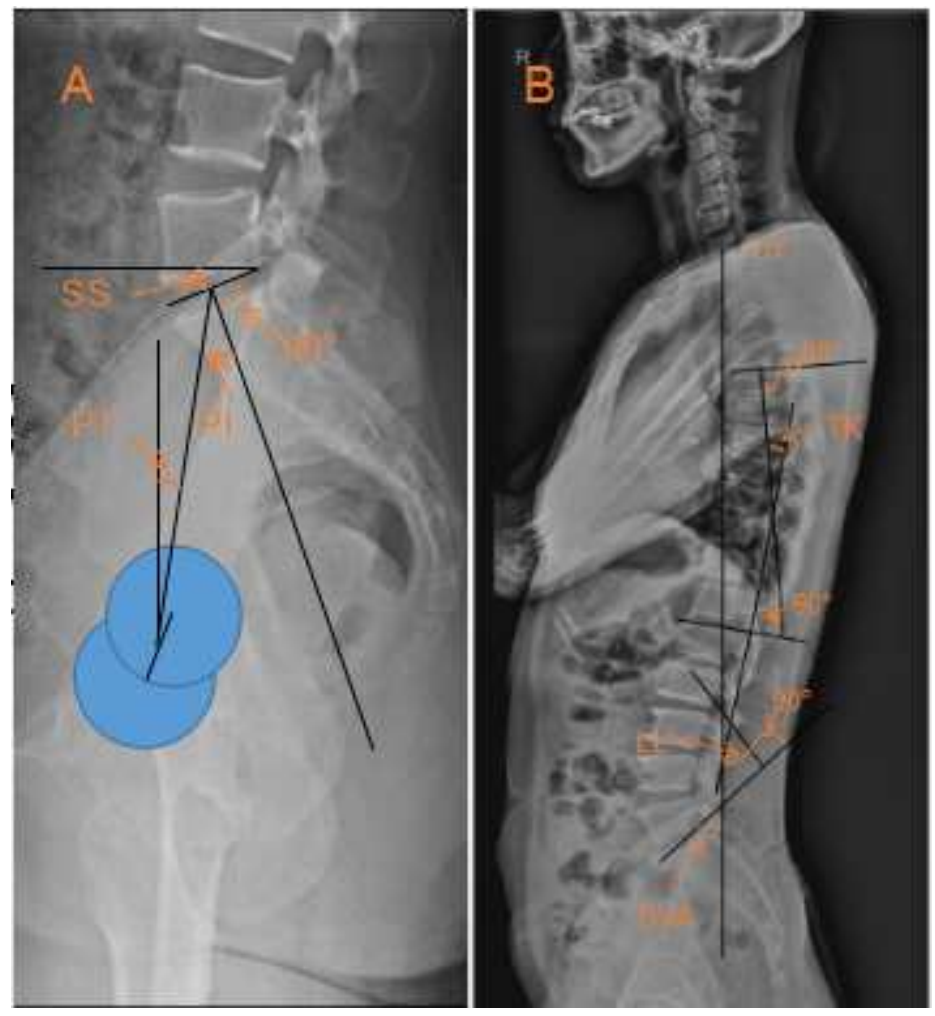

\section{Figure 1}

Ilustration of the conventional radiological parameters of the spine-pelvis on the sagittal plane.The pelvis parameters include pelvic incidence( $\mathrm{PI})$, pelvic tilt(PT), and sacral slope(SS) from picture $\mathrm{A}$. The spine parameters include lumbar lordosis(LL),thoracic kyphosis(TK) and sagittal vertical axis(SVA) from picture B.

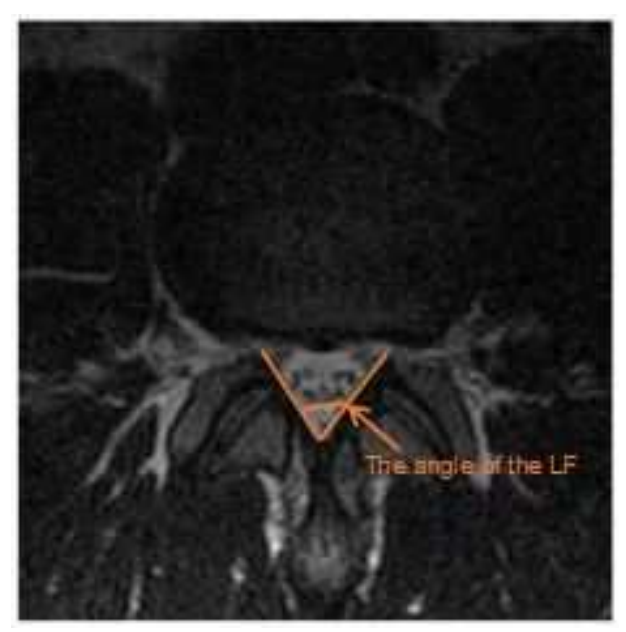

\section{Figure 2}

Illustration of the way to measure the angle of the ligamentum flavum $\varangle \mathrm{LF} \otimes$ of midline level of $L 4 / 5$ intervertebral space. 


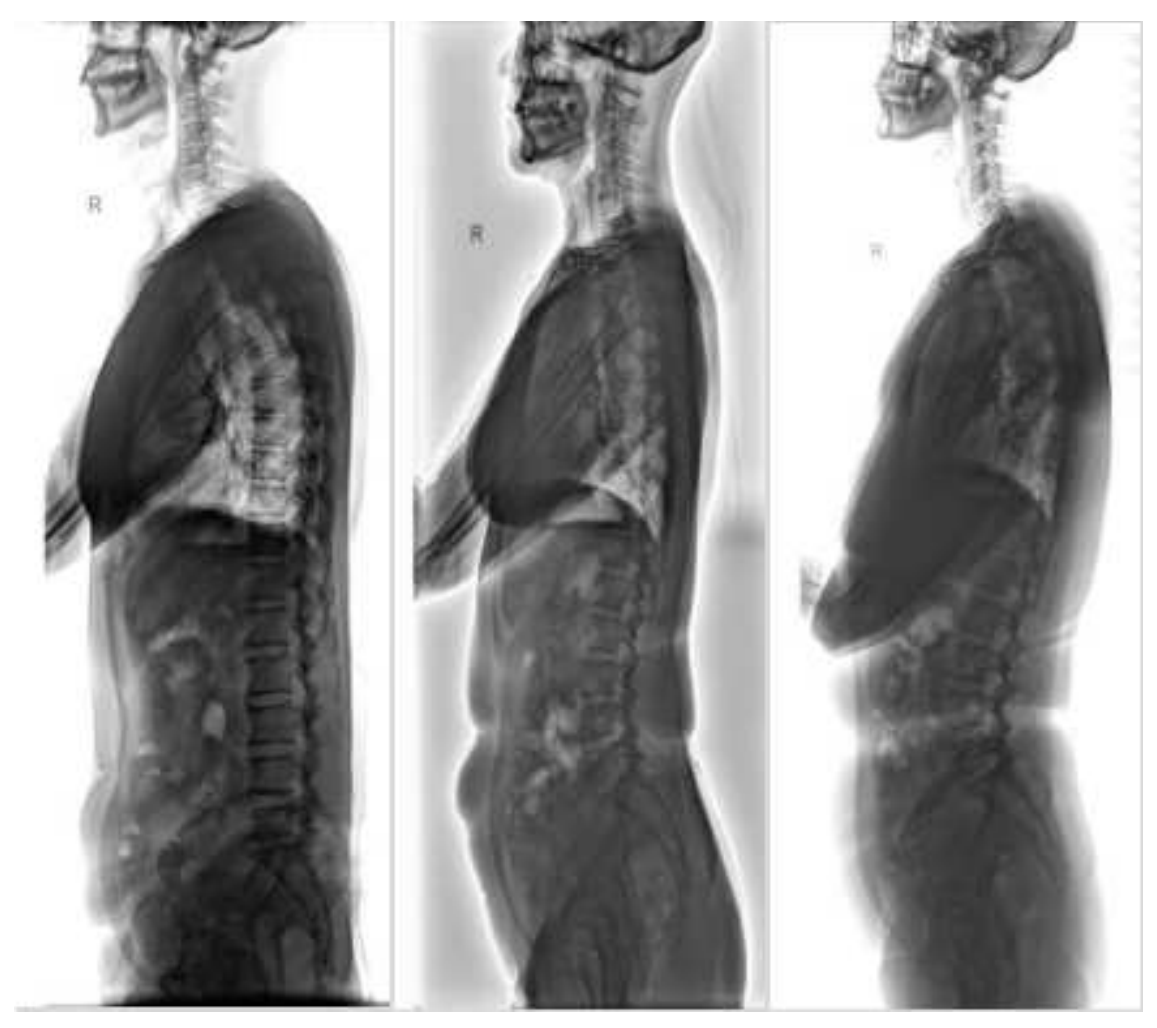

\section{Figure 3}

Comparison of sagittal plane morphology of the CG, AG, NG groups. Arranged from left to right are the whole spine X-rays of patients with congenital lumbar spinal stenosis, acquired lumbar spinal stenosis and normal people. Compared with acquired lumbar spinal stenosis patients group and normal people group, the spine of patients with congenital lumbar spinal stenosis is more vertical and the trunk is more forward. 


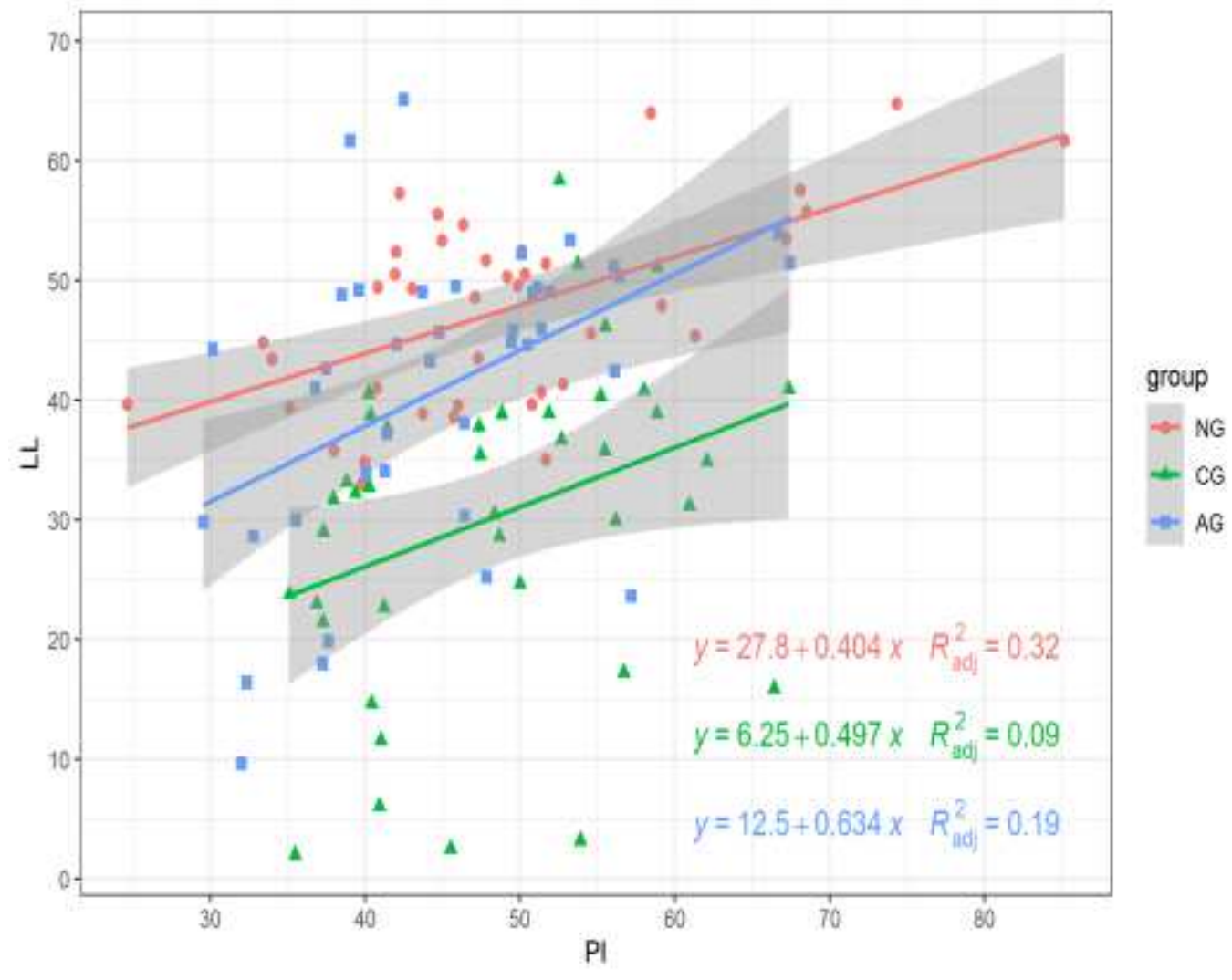

Figure 4

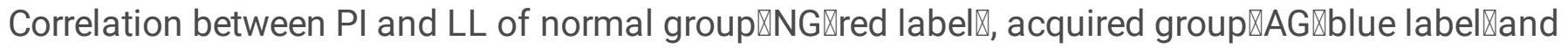

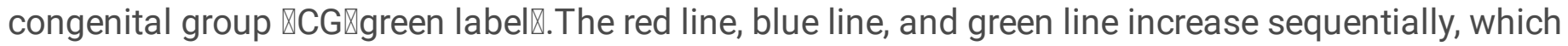
means that the LL/PI trend line of the control group, acquired group, and control group gradually rises. 


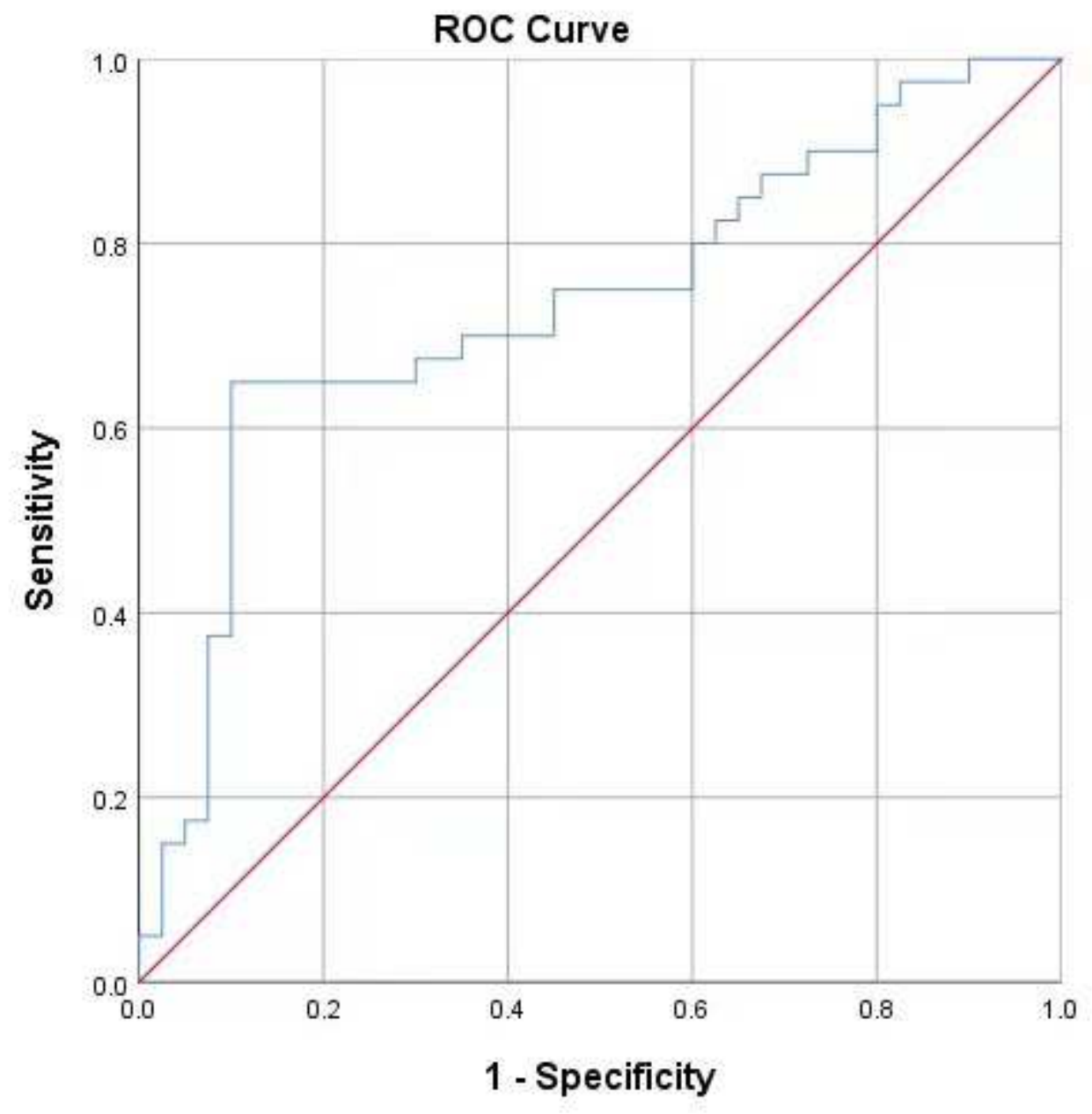

Figure 5

ROC curve of the $L L$ for preliminary screening of patients with congenital lumbar spinal stenosis. The area under the curve (AUC) is :0.735. ROC: receiver operating characteristic. 